\title{
La presenza Italiana in Amazzonia e nel Nordest del Brasile tra Otto e Novecento
}

\author{
Vittorio Cappelli \\ Università della Calabria
}

\section{Introduzione}

Ai margini della grande emigrazione italiana diretta in Brasile - che ha privilegiato, com'è noto, le città e gli Stati del Sud, da Porto Alegre a Vitória, concentrandosi soprattutto a São Paulo e in qualche misura a Rio de Janeiro -, diverse migliaia di emigranti italiani, tra il XIX e il XX secolo, scelsero invece le città del Nordest, da Fortaleza a Salvador de Bahia, e il nord amazônico, da Belém a Manaus. ${ }^{1}$

Gli immigrati italiani giunti nel Nord e nel Nordest del Brasile provenivano quasi sempre dalle province di Salerno, Potenza e Cosenza, più precisamente da un territorio di frontiera dell'Appennino meridionale, posto a cavallo tra Campania, Basilicata e Calabria; un aspro territorio montagnoso che quasi sempre precipita bruscamente sul mar Tirreno, ed è oggi in larga parte compreso tra i due più grandi parchi nazionali italiani, il Parco del Cilento e del Vallo di Diano e il Parco del Pollino. ${ }^{2}$

Erano per lo più artigiani, commercianti e contadini indipendenti, che formavano catene migratorie spontanee. Ciascuna di esse, in partenza da un villaggio o da un paese, si concentrava prevalentemente in una sola città amazônica o "nordestina", con la speranza e il progetto di "fare l'America" attraverso i commerci, l'artigianato e la piccola industria, approfittando delle opportunità offerte dai processi di urbanizzazione, in atto anche nelle capitali del Nordest, e soprattutto approfittando dello strepitoso, per quanto breve, ciclo economico del caucciù (la gomma selvatica della foresta amazônica, a quel tempo fortemente richiesta dall'industria della gomma per la produzione di pneumatici). L'auge dell'esportazione del caucciù (un quarto delle esportazioni brasiliane nel 1910, malgrado il boom del caffe nel sud paulista) ${ }^{3}$ attira in Amazônia non soltanto grandi folle di lavoratori addetti all'estrazione del lattice nelle foreste, provenienti dal Nordest brasiliano (in specie dal Ceará, colpito 


\section{Dôssie Imigração}

dalla siccità), ma anche numerosi immigrati italiani, provenienti dalla Campania, dalla Basilicata e dalla Calabria.

A fianco a queste catene migratorie si registrava anche un'importante presenza italiana nelle arti visive, nella musica, nell'architettura e nell'urbanistica, che si inseriva nei segmenti medio-alti della società locale, stabilendo fruttuosi rapporti col potere politico. Costruttori e architetti adoperavano spesso la massoneria come strumento d'integrazione e talora la stampa e le legazioni diplomatiche, per dare più forza alla loro attività costruttiva, che - tra eclettismo e art nouveau - avrebbe finito col soddisfare nell'oligarchia locale l'esasperato desiderio di imitare e importare anche in Amazônia la mitica belle époque parigina. ${ }^{4}$

Architetti e artisti italiani finiscono dunque col recitare un ruolo di primo piano in uno straordinario laboratorio periferico della modernità, ${ }^{5}$ nel quale, come si vedrà nel testo (e nelle note), l'appartenenza a confessioni religiose protestanti costituisce talvolta, assieme alla massoneria, una variabile culturale di non scarso rilievo.

\section{Italiani in Amazônia}

\section{a. A Manaus}

All'inizio di novembre del 1899, prendeva il largo dal porto di Rio de Janeiro il vapore Iris, della Compagnia Lloyd Brasileiro. Vi era a bordo il grande costruttore italiano Antonio Jannuzzi, che guidava 400 operai, per lo più italiani, alla volta di Manaus, nel cuore dell'Amazônia. ${ }^{6}$

Venticinque anni prima, ossia nel 1874, Jannuzzi, allora giovanissimo scalpellino e capomastro, figlio d'arte, dopo una breve e non entusiasmante esperienza a Montevideo, era emigrato a Rio de Janeiro. Egli era partito, assieme al fratello Giuseppe, da Fuscaldo, un centro che si affaccia sulla costa tirrenica calabrese, culla di un'antica tradizione artigiana di lavorazione della pietra. Era poi divenuto, in non molto tempo, il più importante costruttore della capitale brasiliana, la quale di lì a poco invaghita del mito della belle époque - avrebbe abbandonato del tutto il suo vecchio volto coloniale, per assumere quello di una grande città moderna, modellata sull'esempio della Parigi di Haussman.

Il forte e determinato self made man calabrese era stato chiamato a Manaus dal governatore José Ramalho, per costruirvi il grande ospedale della Santa Casa de Misericórdia, posto alle spalle del sontuoso Teatro 
Amazonas, ancora fresco di cantiere, e del costruendo Palazzo di Giustizia, che sarebbe stato interamente completato tre anni dopo. ${ }^{7} \mathrm{E}$, com'era suo costume, pretese e ottenne di lavorare utilizzando maestranze italiane, per lo più di origine calabrese o addirittura fuscaldese.

Nel giro di pochi giorni fu, dunque, celebrata la posa della prima pietra di un'opera molto impegnativa per il governo dell'Amazonas dal punto di vista finanziario. Dell'evento diede notizia alla stampa della capitale, da Manaus, Vincenzo Scirchio, braccio destro di Jannuzzi. ${ }^{8}$ Ma le cose non andarono per il verso giusto: "dopo mille peripezie e disgrazie, durate per lo spazio di due anni", Jannuzzi dovette fare i conti con l'ostilità al progetto del nuovo governatore dell'Amazonas Silverio Nery. Di conseguenza, chiese e ottenne per la rescissione del contratto un cospicuo risarcimento dal governo amazônico. Per questa grande opera incompiuta - che, tra l'altro, fece anche non poche vittime tra i lavoratori -, ottenne un indennizzo di 5 milioni di lire. ${ }^{9}$

Il nostro costruttore pare abbia gestito l'intera operazione da Rio, dove per motivi di salute era rientrato due mesi dopo l'inizio dei lavori. ${ }^{10} \mathrm{E}$ del tutto plausibile immaginare che a Manaus, invece, fosse rimasto, dopo esservi giunto presumibilmente con lo stesso vapore Iris di Jannuzzi, l'ingegnere Filinto Santoro, un altro fuscaldese, amico di famiglia dei Jannuzzi. L'ingegner Filinto si era aggregato ai fratelli Jannuzzi a Rio nel 1889. E Antonio Jannuzzi lo aveva subito messo a capo dell'ufficio tecnico della sua Companhia Evoneas Fluminense, ideata per la costruzione di case operaie.

Tra le famiglie Jannuzzi e Santoro di Fuscaldo c'erano molteplici rapporti, rinvigoriti dalle esperienze migratorie in Brasile di molti loro esponenti. Antonio Jannuzzi, il capostipite di questa saga migratoria fuscaldese, prima di partire per le Americhe col fratello Giuseppe, da giovane scalpellino aveva appreso il disegno architettonico dalle lezioni del pittore Giovan Battista Santoro, zio dell'ingegnere Filinto e dei fratelli di questi (il pittore Rubens, il medico Aleardo, il musicista Giotto, Attilio...; tutti, ad eccezione di Rubens, emigrati in Brasile, ma preceduti nel 1883 da un loro cugino, il pittore Rosalbino). Le affinità tra le due famiglie sono confermate dalla comune appartenenza alla Chiesa Valdese, che ha antiche tradizioni nella zona di Fuscaldo, Guardia Piemontese, Acquappesa, Montalto Uffugo, San Sisto dei Valdesi, ecc.La cultura protestante viene poi rielaborata da Antonio Jannuzzi in Brasile, dove egli si fa pioniere della Chiesa Presbiteriana, di spiccata ispirazione calvinista, alla quale 
avevano aderito verso la fine dell'Ottocento i Valdesi italiani emigrati negli USA. ${ }^{11}$

Filinto Santoro, nei primi anni della sua lunghissima avventura brasiliana, si era molto impegnato anche nel giornalismo italiano della capitale ed era ben presto entrato in contatto anche col Presidente della Repubblica Floriano Peixoto, avviando una brillante carriera, ma senza uscire, per il momento, dall'orbita degli interessi e dei successi della famiglia Jannuzzi e dei numerosissimi fuscaldesi di Rio, organizzati dal 1886 nella Società Operaia Fuscaldese di Mutuo Soccorso, presieduta da Giuseppe Jannuzzi, mentre il fratello maggiore Antonio dirigeva la più antica Società Italiana di Beneficienza. ${ }^{12}$ Lo stesso Antonio si segnalava anche come benefattore della Chiesa Presbiteriana e ricopriva alte cariche nella massoneria. ${ }^{13}$

Le cose cambiano in seguito al viaggio a Manaus del 1899. Mentre Antonio Jannuzzi rientra per sempre a Rio, dove celebrerà di lì a poco $\mathrm{i}$ più grandi successi della sua carriera nella costruzione dell' Avenida Central, ${ }^{14}$ il governatore dello Stato dell'Amazonas, José Ramalho, chiede a Filinto Santoro una consulenza circa la possibilità di ristrutturare e adeguare il Palazzo di Giustizia della città. L'ingegnere esclude la possibilità di intervenire con una ristrutturazione dell'esistente e propone invece un progetto grandioso, ${ }^{15}$ che vorrebbe competere col sontuoso Teatro Amazonas (per inciso, il salone nobile, ossia lo spazio interno più lussuoso, del celebre e un po' folle teatro di Manaus, con i suoi dipinti, i vetri di Murano, gli stucchi, il parquet in legno con elaborati disegni geometrici, è opera in gran parte dell'artista italiano Domenico De Angelis). ${ }^{16}$

Ramalho accetta il progetto del nuovo Palazzo del Governo e Santoro dà il via ai lavori, per i quali costituisce una squadra tecnica e operaia composta da circa 300 italiani, affidando l'incarico della direzione amministrativa dei lavori al suo fratello musicista Giotto. I modelli di riferimento dell'idea progettuale d'impronta eclettica sono i Palazzi di Giustizia di Bruxelles (opera di Polaert) e di Roma (Calderini), nonché il "Vittoriano", progettato a Roma per il re Vittorio Emanuele II (Sacconi).

Nel maggio del 1900 si dà inizio ai lavori, ma un anno dopo il nuovo governatore, Silvério José Nery, dichiara tutta la sua contrarietà alla prosecuzione dell'opera, giudicata troppo onerosa, e l'impresa si arenerà senza rimedio. Di conseguenza, Santoro rimane a Manaus solo il tempo necessario per costruire una palazzina, per riformare il vecchio mercato cittadino e per progettare la nuova Igreja dos Remédios, in stile classico 
eclettizzato, la quale va ad aggiungersi alla più antica Igreja de São Sebastiāo (1888), dove, sotto la direzione del frate missionario francescano Gesualdo Machetti, aveva lavorato il costruttore Alberto Grossi e aveva dipinto opere assai pregevoli il pittore Domenico De Angelis. ${ }^{17}$

L'ingegnere Santoro, nel 1903, si dirige a Belém, lasciando però a Manaus i suoi fratelli Attilio e Giotto. Quest'ultimo, in specie, lascia traccia di sé: diventerà amministratore del porto di Manaus e avrà 15 figli, uno dei quali è il grande musicista brasiliano Claudio Santoro (Manaus, 1919-Brasilia, 1989), cui oggi è dedicato il moderno teatro nazionale di Brasilia, progettato da Oscar Niemeyer. ${ }^{18}$

Per qualche tempo ancora seguiterà a Manaus la belle époque equatoriale del caucciù. I coronéis della gomma selvatica, a differenza del tradizionalismo aristocratico dei fazendeiros del caffe nel sud fluminense e paulista, sono tentati e affascinati dall'internazionalismo della belle époque e dai suoi entusiasmi franchi e irresponsabili. Ma naturalmente - ci ricorda Márcio Souza - dietro il volto trionfante del caucciù, costituito dal rigoglioso e moderno paesaggio urbano di Manaus, si celano, nascosti nell'infinito groviglio dei fiumi e delle foreste, gli orrori dello sfruttamento selvaggio dei raccoglitori del latex, l'oro bianco dell'Amazônia. ${ }^{19}$ E nella stessa città di Manaus, all'entusiasmo e al lusso ostentato della élite fanno da contrappunto la prostituzione, il vagabondaggio e le tensioni sociali. ${ }^{20}$

Va precisato, peraltro, che la presenza degli italiani a Manaus non si limita soltanto a quella artistica delle architetture di Santoro o delle pitture di De Angelis. Varie testimonianze ci dicono che agli inizi del Novecento vivono in città tra i 1.500 e i 2.000 immigrati italiani, non pochi dei quali attivi nell'artigianato, nei commerci e nella piccola industria. ${ }^{21} \mathrm{Si}$ tratta di una presenza notevole, se si considera che Manaus agli inizi del Novecento ha circa 50.000 abitanti, tra i quali moltissimi sono gli stranieri di varie nazionalità.

Dal 1897 al 1904 gli immigrati italiani possono approfittare della linea di navigazione Genova-Manaus della Compagnia Ligure-Brasiliana, attivata dall'armatore genovese Giulio Gavotti. Nelle navi italiane dirette in Amazônia, dunque, spesso si mescolano gli immigrati e le compagnie liriche italiane chiamate ad esibirsi nel Teatro Amazonas. Segue la stessa rotta il capitano di fregata Gregorio Ronca, nel 1904, risalendo il Rio Amazonas con la sua nave oceanica Dogali, su incarico del governo italiano, per avviare relazioni commerciali e allacciare rapporti con gli immigrati italiani in Amazônia. A Manaus, Ronca è impressionato dalla 
modernità della città, dotata di strade larghe e diritte, percorsa dai tram, illuminata dalla luce elettrica e dominata dalle guglie della cattedrale e dalla grande cupola del teatro. In città, gli dicono, vivono circa duemila italiani, molti dei quali però vivono in condizioni difficili, addetti a lavori umili, come il facchinaggio nel porto fluviale. E Ronca sospetta pure che un certo numero di italiani si sia disperso nelle seringueiras, tra i raccoglitori del caucciù. Ma una minoranza di operai pare che guadagni bene e una settantina di commercianti e impiegati sono in discrete condizioni. $^{22}$

Il giornalista Alfredo Cusano, nel 1911, offre informazioni ancora più precise: i $2 / 3$ degli italiani sono manovali, facchini e lustrascarpe, $1 / 3$ è costituito da piccoli industriali, piccoli commercianti e impiegati di commercio. Molti arricchiscono, ma gli stessi facchini e lustrascarpe guadagnano 15-20 lire al giorno (si consideri che nel 1906, in Calabria e Basilicata, i salari agricoli, notevolmente aumentati proprio in conseguenza dell'emigrazione, non superano nel momento migliore, che è quello della mietitura, le 4 lire al giorno ${ }^{23}$. Ci sono, infine, tra gli italiani di Manaus grandi ditte commerciali come le gioiellerie e le oreficerie di Secondino Lora, Benedetto Dalia, Poli \& C., nonché i negozi di stoffe e indumenti di Marinelli e Vulcani. ${ }^{24}$

È interessante osservare che la moda europea circoli a Manaus grazie, principalmente, all'iniziativa di commercianti italiani: la camiceria Old England è proprietà di José P. Vulcani; la casa di moda Au Bon Marché è proprietà dei signori Lifsitch \& Russo; titolari di importanti sartorie cittadine sono Madame Marietta, nonché Adolfina \& C.ia, successores de Madame Marie; le migliori flanelle inglesi, i più eleganti tailleur, os figurinos de Londres e Paris sono offerti dalla sartoria Poli \& Companhia. ${ }^{25}$

Lo stesso Cusano sottolinea poi che gli italiani non partecipano al commercio del caucciù e che "appena formano un piccolo capitale lo mandano in Italia", dove tutti desiderano tornare. ${ }^{26} \mathrm{Ma}$, se è vero che questo sembrerebbe essere il progetto della maggioranza degli immigrati, un altro autore, Giovanni Bonacci, osserva nel 1920 che gli italiani in Amazônia "non sono molti, ma certo in numero ben superiore a quanto si soglia dire". Lo stesso Bonacci precisa poi: "A Manaos e a Parà non mancano esportatori nostri connazionali. Ne ho conosciuti alcuni che vivono colà da un decennio, vi hanno realizzato lucri non indifferenti, vi godono perfetta salute e sono arcicontenti di essersi una buona volta decisi a lasciare i magri impieghi che occupavano in Italia". ${ }^{27}$ 
Infine, da una rassegna sugli italiani nel Nord del Brasile, pubblicata nel 1931-32, si ricava che per non pochi di essi l'immigrazione era diventata definitiva. ${ }^{28}$ La permanenza di molti a Manaus negli anni Trenta, quando l'auge del caucciù e le ricchezze spropositate dei protagonisti di quel ciclo economico erano ormai un ricordo lontano, documenta il radicamento di parte degli immigrati italiani, che non abbandonano l'Amazônia neppure in un momento di crisi assai grave.

Leggendo la citata rassegna sugli italiani di Manaus, si apprende anche che il nucleo più consistente degli immigrati proveniva da Castelluccio Inferiore, un paesino della Basilicata, dove ancora oggi si possono osservare ampie tracce di questa esperienza migratoria: la strada principale del paese è ancora conosciuta come "la via dei brasiliani", costellata, all'ingresso delle case principali, di piccoli portali in pietra, datati e siglati con le iniziali dei "brasiliani" tornati in patria per investirvi i loro risparmi. I castelluccesi di Manaus, inoltre, donarono alla chiesa matrice del loro paese, nel 1910, un grande orologio meccanico e delle campane, da installare sul campanile. ${ }^{29}$

Diversi immigrati castelluccesi, negli anni Trenta, gestivano laboratori di orologeria e oreficeria; altri avevano avviato marmerie, lattonerie e calzolerie. A fianco a loro, la rassegna del '32 rappresentava anche l'attività di immigrati originari di Casalbuono, Sapri, Maratea, San Costantino di Rivello, Trecchina, Latronico, Rotonda e Laino Borgo, tutti paesi di frontiera tra Campania, Basilicata e Calabria, nessuno dei quali è molto lontano da Castelluccio. A fianco ad essi si registrava, inoltre, la presenza religiosa dei Padri Cappuccini, provenienti dall'Umbria di San Francesco d'Assisi. ${ }^{30}$

Non va dimenticato, infine, tra gli italiani di Manaus, un personaggio straordinario come l'emiliano Ermanno Stradelli (Borgotaro, Parma, 1852Manaus, 1926), grande esploratore ed etnologo, che visse in Amazônia per più di quarant'anni, facendone la sua patria d'adozione, fino alla morte, intervenuta nel lebbrosario Umirizal. Nel 1897, Stradelli fece anche un tentativo di fondare una compagnia italo-brasiliana per l'estrazione del caucciù, facendosi mediatore tra la nuova élite amazônica e l'Italia. Ma la sua iniziativa fallì, come sarebbe accaduto nel 1904 alla compagnia di navigazione ligure-brasiliana di Gavotti, contestualmente al viaggio del capitano Ronca. ${ }^{31}$

Non ci saranno in seguito altri tentativi miranti a costruire una presenza economica significativa dell'Italia in Amazônia, a fronte di una solida e indiscussa egemonia britannica. Si consideri che a fine Ottocento ben 20 
vapori britannici sono impiegati per il trasporto dei prodotti amazonici sulle piazze di New York, Liverpool, Le Havre, Amburgo, Lisbona e Porto. E, a mo' di comparazione, si pensi che nel 1901 ben 229 vapori britannici entravano nel porto di Belém e 101 nel porto fluviale di Manaus, a fronte, rispettivamente, di 19 e 9 vapori italiani. ${ }^{32}$

\section{b. A Óbidos e Santarém}

Óbidos e Santarém, con i loro approdi fluviali, posti più o meno a metà strada tra Manaus e Belém, lungo il Rio Amazonas, sono snodi commerciali importanti, tra Otto e Novecento, non solo per il caucciù ma anche per il cacao, per le castanhas do parà e per il pirarucu. ${ }^{33}$

In queste due cittadine vivono numerosi italiani, quasi tutti provenienti dal paesino lucano di San Costantino di Rivello (Potenza). Il pioniere di questa catena migratoria, un calderaio, giunse nel 1888 e avviò a Santarém un'attività commerciale, pienamente operante ancora negli anni Trenta del nuovo secolo come ditta "Mileo \& Calderaro", attiva nel commercio di legnami, ma anche di cacao, bestiame, riso e cotone. ${ }^{34}$

Nel 1904, quando nel territorio di queste due cittadine fluviali abitano complessivamente non più di trentamila abitanti, il capitano Ronca, nel corso del suo viaggio in Amazônia, incontra quasi un centinaio di italiani. Essi gestiscono, tra Óbidos e Santarém, una ventina di case commerciali che fanno ottimi affari. ${ }^{35}$

Da un accuratissimo inventario del patrimonio architettonico di Óbidos emerge con forza, inoltre, la presenza italiana nella cittadina e anche un curioso e significativo dettaglio: la residenza di Antonio Calderaro, originario di San Costantino di Rivello, è anche sede della Antiga Maçonaria e successivamente della Igreja Batista. ${ }^{36}$

A Santarém vi è anche un'eccentrica famiglia di commercianti, i Peluso, che trattano merci assai particolari, come i cappelli di Firenze e i coralli napoletani. Per giunta, la signora Marietta Mattera, consorte del capofamiglia Domenico Peluso, mette in piedi anche una piccola scuola di musica, fin quando, chiuso il ciclo del caucciù, nel 1923, non si trasferiranno tutti a São Paulo, dove potranno con molto più agio coltivare la passione per la musica. ${ }^{37}$

Un'altra impresa italiana ancora attiva negli anni Trenta in questa regione, ma molto più vicino a Belém, è quella di Garibaldi Parente, figlio di Nicola Maria, un immigrato di Marsico Nuovo, in Basilicata, che aveva 
percorso tutto il Brasile dal 1876, come pioniere nomade del cinema, fotografo, dentista, calzolaio, chimico... Nicola Maria si era stabilito infine, intorno al 1896, ad Abaeté (oggi Abaetetuba), alla foce del Rio Tocantins, dove morì nel 1911, lasciando la sua ditta commerciale al figlio Garibaldi. ${ }^{38}$

\section{c. A Belém}

La città di Belém, dal punto di vista amministrativo è la capitale dello Stato del Pará, ma dal punto di vista geografico è la porta d'ingresso all'Amazônia, posta com'è a fianco alla grande isola di Marajó, che si frappone tra il Rio Amazonas e l'Oceano Atlantico. Dal punto di vista economico, Belém è il principale snodo portuale della navigazione e dei commerci tra l'Amazônia e il resto del mondo.

Assai vistosa è la presenza culturale italiana in città, che risale addirittura al XVIII secolo. Lo dicono ancora oggi le architetture neoclassiche di Giuseppe Antonio Landi (Bologna, 1713-Belém, 1791), ancor oggi poco conosciuto in Italia ma celebratissimo a Belém. ${ }^{39}$ Nella seconda metà del XIX secolo, l'architettura italiana è di nuovo celebrata con il Teatro da Paz (1878), che s'ispira visibilmente al Teatro alla Scala di Milano. Gli interni sono decorati dal pittore Domenico De Angelis, lo stesso che in seguito lavorerà, come si è detto, alla Igreja de São Sebastiāo (1888) di Manaus e al Teatro Amazonas (1897), guidando una specie di "missione artistica" italiana che è emanazione della romana Accademia di San Luca. ${ }^{40}$

In questa città - come e forse più che a Manaus -, sull'onda del boom del caucciù, esplode una sorta di delirio culturale francesizzante. Il desiderio di riprodurre gli splendori della belle époque conduce alla formulazione del sogno di una Paris n'América (non è un caso che sia questo il nome di un grande magazzino, aperto in un sontuoso palazzotto art nouveau). L'élite economica, arricchitasi smisuratamente col commercio della gomma selvatica, manifesta comportamenti grotteschi, come quello di vestirsi in modo assolutamente incongruo a quella latitudine, indossando camicie inamidate, abiti di lana e addirittura soprabiti e tuba, per esibire status e ricchezza. Gli esponenti dell'oligarchia del caucciù, non solo mandano i figli a studiare in Europa, ma acquisiscono ed esibiscono abitudini a dir poco eccentriche, come quella di mandare a lavare i panni a Londra o far rilegare i libri a Parigi. L'ubriacatura francesizzante è talmente incontrollata che le prostitute ebree provenienti dall'est europeo, note a Rio e São Paulo con l'appellativo non esattamente lusinghiero di "polacche", in Amazônia, invece, a Belém come a Manaus, diventano come d'incanto raffinate "francesi". ${ }^{41}$ 


\section{Dôssie Imigração}

Con tutta evidenza, il carattere brusco e repentino del mutamento economico e sociale non consente una matura assimilazione di nuovi abiti mentali. I timidi e tradizionali costumi provinciali - sia a Manaus che a Belém - sono frantumati dal cosmopolitismo introdotto dalla fluttuante popolazione straniera e dai brasiliani di altre regioni, che destrutturano la fragile società locale, esponendola ad una violenta irruzione di modernità. ${ }^{42}$

Di tal fatta è il contesto nel quale, dal 1903 al 1913, l'architetto Filinto Santoro, abbandonata Manaus, lavora freneticamente, costruendo villini per i principali uomini politici di Belém e numerosi edifici pubblici, approfittando di una congiuntura davvero ghiotta. Si calcola, infatti, che nel solo 1909, quando la città ha 200.000 abitanti, si siano edificate 14.000 nuove costruzioni. ${ }^{43}$

Tra i progetti di Filinto Santoro, di particolare interesse è il Palacete Montenegro (1903), costruito per l'allora governatore del Pará Augusto Montenegro. L'edificio presenta persistenze stilistiche neoclassiche consone al ruolo pubblico del governatore -, combinate però, come sottolinea Jussara Derenji, con dettagli interni ed esterni di "inusitata raffinatezza". ${ }^{44}$ L'esecuzione dei lavori è affidata al capomastro Luigi Bisi, un immigrato italiano che accompagna Santoro in tutti i suoi lavori di Belém. I materiali necessari alla realizzazione del Palacete sono interamente importati dall'Italia, tramite la ditta Frisoni di Genova. Così avverrà per tutte le altre costruzioni di Santoro a Belém, malgrado che la chiusura della linea di navigazione Genova-Manaus, obblighi, dal 1905, ad un laborioso e costoso trasbordo dei materiali a Lisbona. ${ }^{45}$

Tra le altre numerose costruzioni realizzate da Santoro a Belém, si segnalano la residenza del senatore Virgilio Sampaio (1903), l'edificio del giornale A Província do Pará (1904) e il Colegio Gentil Bittencourt (1906). ${ }^{46}$

Ma l'opera pubblica più importante costruita da Santoro in Amazônia è probabilmente il Mercado São Braz (1911) di Belém, una struttura piuttosto imponente, di equilibrata composizione, che presenta una facciata principale lunga 100 metri e due facciate laterali di 46 metri, lungo le quali si svolgono due portici, coronati da eleganti colonnati ionici. L'uso dei materiali - ancora una volta interamente importati dall'Italia - , non risparmiando nulla nell'utilizzo dei marmi negli interni, dà un tono lussuoso all'insieme, pur trattandosi di una costruzione destinata ad una frequentazione popolare. Il costo complessivo dell'edificio ammonta a tre milioni e mezzo di lire. ${ }^{47}$ 
Nei suoi dieci anni di permanenza a Belém, Filinto Santoro ricopre anche l'incarico di Console d'Italia e si occupa, dunque, sistematicamente della nutrita colonia peninsulare, che Alfredo Cusano fa ammontare a circa 1.200 persone..$^{48}$ La comunità italiana è caratterizzata dalla netta prevalenza di commercianti-artigiani, attivi soprattutto nella produzione e nel commercio di generi alimentari, materiali da costruzione, gioielli ed orologi, ma soprattutto abiti e scarpe. Sono numerose in particolare le sartorie e le calzolerie. Si tratta quasi sempre di imprese a carattere familiare, in cui sono occupati fratelli, figli, generi, nipoti, cugini, ecc.; che però pervengono talvolta ad una dimensione industriale. È questo il caso della fabbrica di scarpe Bôa Fama, di Nicola Conte, originario di Castelluccio Inferiore (Potenza), che nel 1912 utilizza macchine elettriche e conta 200 dipendenti (l'azienda ha un deposito anche a Manaus). Naturalmente si tratta di un caso limite. Molto più spesso i calzaturifici hanno dimensioni più modeste, come nel caso di Francesco Cerbino, di Laino Borgo (Cosenza), che nel 1912 fonda una calzoleria nella quale lavorano sette suoi figli, o in quello di Francesco Libonati, di Rotonda (Potenza), che aprirà un'altra calzoleria nel $1924 .{ }^{49}$

Un'altra attività, assai più vicina agli interessi del console-ingegnere Filinto Santoro, è quella degli addetti alle costruzioni. In quest'ambito, una figura di particolare interesse è quella di Antonio Vita, un capomastro, giunto nel 1901 a Belém da Casalbuono, un paesino campano al confine con la Basilicata. Egli dirige per un quarto di secolo i lavori di costruzione della Basilica di Nossa Senhora de Nazaré (1909), voluta dai Padri Barnabiti, presenti a Belém dal 1903, e progettata in Italia da Gino Coppedè, un grande protagonista dell'eclettismo italiano, che nell'ideazione si è ispirato esplicitamente alla chiesa romana di San Paolo fuori le mura.

Antonio Vita, nel 1927, si mette in proprio con una sua ditta di costruzioni, testimoniando così, assieme a molti altri, una continuità di quest'immigrazione italiana, che si protrae ben oltre i limiti cronologici del ciclo economico del caucciù. ${ }^{50}$

Non va trascurata, infine, un'altra presenza artistica italiana, di grande rilievo nella belle époque del caucciù: quella musicale, che non si riduce alla, pur importante, circolazione delle compagnie liriche italiane chiamate ad esibirsi al Teatro da Paz di Belém e al Teatro Amazonas di Manaus (a questo proposito, sarebbe troppo lungo esporre l'elenco delle celebrità coinvolte: da Enrico Bernardi - che nel 1880 dirige a Belém l'Ernani di Verdi - alla compagnia lirica italiana che nel 1897 inaugura il Teatro 
Amazonas con La Gioconda di Amilcare Ponchielli, alle più tarde esibizioni di Tito Schipa e Beniamino Gigli).

A Belém - ed è forse solo l'esempio più macroscopico - vive per quasi mezzo secolo il maestro-compositore Ettore Bosio (Vicenza, 1862-Belém, 1936)..$^{51}$

Formatosi presso il Liceo Musicale di Bologna, Bosio, già nel 1888, decide di recarsi in Brasile. Dopo un breve soggiorno a Rio e a São Paulo, sceglie di stabilirsi a Belém. Qui, nel Teatro da Paz, viene rappresentata, nel 1891, la sua opera $O$ Duque de Viseu (su libretto portoghese). Un'altra sua opera (su libretto italiano) è l'Ideale, rappresentata una prima volta a Bologna e poi a Belém nel 1900. La produzione musicale di Bosio è molto variegata. Egli è autore anche di composizioni sinfoniche per orchestra, romanze, barcarole, ecc.; e si segnala in particolare per il suo Samba do Costa, un samba sinfonico che mostra il largo e profondo assorbimento della cultura amazônica da parte del compositore italiano. ${ }^{52}$

Dal 1929, Bosio dirige l'Instituto Carlos Gomes, un conservatorio che era nato nel 1895 con la direzione dello stesso Carlos Gomes, il maggior musicista brasiliano del XIX secolo. Negli anni Trenta, fino alla sua scomparsa, Bosio viene considerato ormai un'autorità culturale indiscussa e in qualche modo è anche un vanto della comunità italiana di Belém. ${ }^{53}$

\section{Italiani nel Nordest. Da Fortaleza a Salvador de Bahia}

Come si è anticipato nell' introduzione, gli immigrati italiani sono attratti non solo dalle opportunità offerte dai commerci e dalla crescita degli spazi urbani nell'Amazônia del caucciù, ma anche dai processi di urbanizzazione in atto, tra Otto e Novecento, nelle città del Nordest, da Fortaleza fino a Salvador de Bahia, che danno un contributo non trascurabile alla creazione di una rete urbana moderna in Brasile. Si consideri che le città con più di centomila abitanti, che erano nel 1870 soltanto sei, diventano tredici nel 1900 e trentacinque nel 1930, mentre si moltiplicano i municipi con più di diecimila abitanti (più di mille agli inizi del Novecento). ${ }^{54} \mathrm{E}$ tutto ciò non riguarda solo il Sud del Brasile, che è alla testa dello sviluppo economico, ma anche le lontane città "nordestine".

In questa seconda parte del nostro lavoro descriviamo rapidamente la presenza sociale e culturale degli immigrati italiani nella macroregione del Nordest. 


\section{a. A Fortaleza}

Da Tortora, un paesino calabrese situato sulla costa tirrenica, al confine con la Basilicata, parte la principale catena migratoria diretta a Fortaleza. Un esponente di successo di questa catena migratoria è Francesco D’Angelo, ricco importatore di calzature, che nel 1932 pubblicizza la sua attività commerciale esibendo l'immagine della "Casa Veneza", l'edificio in art déco che ospita la sua attività commerciale, e un altro edificio di sua proprietà nella centralissima Rua Floriano Peixoto. ${ }^{55}$

La specialità degli immigrati calabresi di Tortora sembra essere quella delle tintorie e lavanderie a secco. In quest'attività compare anche qualche piccolo protagonismo femminile. Anna Cunto, giunta in Brasile nel 1895, ha aperto una lavanderia elettrica a Fortaleza nel 1919, ancora attiva negli anni Trenta. Ma emergono anche altre attività: il commercio di automobili e macchine da cucire, empori di prodotti italiani, articoli di sartoria e generi alimentari 56

Una ditta, fondata nel 1880, per l'importazione di commestibili e mercerie dall'Europa mostra la precocità di questa immigrazione, confermata dall'arrivo ancor più precoce del calabrese Carlo Mesiano, che nel 1870 apre una gioielleria. Suo figlio Henrique sarà poi un pioniere del cinema a Fortaleza, fondando il Cinema Rio Branco (1909), assieme all'altro calabrese Roberto Muratori, orologiaio. ${ }^{57}$

Un altro pioniere del cinema a Fortaleza è il napoletano Vittorio Di Maio, che, dopo aver introdotto il cinema a Rio de Janeiro (1896) e a São Paulo (1899-1902), decide di aprire a Fortaleza il Cinema Art Nouveau (1908-1914). ${ }^{58}$ Anche in questo caso, dunque, come a Belém e altrove, è un italiano a soddisfare l'infatuazione per Parigi, tipica della belle époque brasiliana.

\section{b. A Natal}

L'agente consolare italiano a Natal, dal 1919, è un immigrato di Battipaglia (Salerno), che si dedica al commercio internazionale: esporta gusci di tartaruga e importa dall'Europa vini e liquori.

Alcuni immigrati italiani, giunti in Brasile negli anni Ottanta dell'Ottocento, scelgono di fare i commercianti a Natal agli inizi del nuovo secolo. E negli anni Venti del Novecento continuano a recarsi a Natal, da altri luoghi del Brasile, degli immigranti italiani: è il caso della sartoria "Amazonas", aperta da un immigrato originario di Maratea (Potenza) e 
della lavanderia "Savoia", di un calabrese di Tortora, avviate dopo un soggiorno di questi stessi immigrati in Pernambuco. ${ }^{59}$

Anche nella piccola Natal, l'immigrazione italiana ha qualche risvolto culturale: nel 1910 il governatore del Rio Grande do Norte chiama come professore di musica il violoncellista Tommaso Barbini, di Faenza; e nel 1929, il sindaco Omar O'Grady, d'origine e formazione nordamericana, affida il "Piano di Sistemazione per l'Espansione Urbana di Natal" all'architetto italiano Giacomo Palumbo, già impegnato precedentemente in progetti pubblici a Recife. ${ }^{60}$

\section{c. A João Pessoa}

Gli italiani immigrati a João Pessoa hanno una loro associazione dal 1890. Quarant'anni dopo, gli italiani si segnalano ancora per la loro iniziativa nei commerci e nelle costruzioni. L'agente consolare, negli anni Trenta, è Vicente Cozza, un commerciante di tessuti e profumi. La sartoria sembra essere l'attività prediletta da diversi italiani, ma certamente le attività più redditizie, negli anni Venti e Trenta, sono le costruzioni, nelle quali sono impegnati con successo due architetti: Ermenegildo Di Lascio, Presidente della Società Italiana di Beneficenza e Maestro Venerabile della Loja Maçônica "Branca Dias", e Giovanni Gioia, segretario del Fascio all'estero di João Pessoa. In quest'ambito di attività, si segnala l'Associação Comercial do Estado da Paraíba, un edificio realizzato da Di Lascio nel 1919. ${ }^{61}$

\section{d. A Recife}

Tra i non pochi italiani giunti a Recife, in genere artigiani e piccoli commercianti, alcuni iniziano come mascates (venditori ambulanti). Numerosi sono gli artigiani specializzati nella lavorazione dei metalli (calderai, lattonieri, fonditori), attratti dalle usinas per la lavorazione dello zucchero. E non è infrequente neppure l'accesso a una dimensione industriale (metallurgia, tessile, calzature, bibite...). ${ }^{62}$

Il gruppo più consistente degli italiani di Recife proviene da Trecchina, un paese della Basilicata caratterizzato da una robusta tradizione artigiana, nella quale spiccano i calderai e gli orefici. Gli immigrati trecchinesi svolgono attività commerciali e industriali, come nel caso dei fratelli Lucchesi e dei fratelli Vita. Questi ultimi, giunti in Brasile nel 1880, hanno fabbriche di bibite (gassose, acque minerali, guaranà, etc.), sia a Recife che a Salvador. Non pochi si dedicano alla metallurgia e aprono fonderie. ${ }^{63}$ 
Altri italiani hanno fabbriche di calzature, di cioccolata e pasta. Altri ancora importano macchine da scrivere Olivetti. Altri aprono calzolerie, sartorie e lavanderie.

Qualche volta il successo economico ha sbocchi culturali e artistici: è il caso della famiglia di Vincenzo La Greca, immigrato da Santa Domenica Talao, un paese calabrese posto quasi al confine con la Basilicata. Egli possiede uno zuccherificio a Palmares. Uno dei suoi dodici figli, Vicente Murillo La Greca (1898-1985), formatosi a Rio, diventerà un affermato pittore, esperto anche nella tecnica dell'affresco, dopo aver studiato e vissuto a Roma e a Santa Domenica Talao per diversi anni (1919-25; 1936-39). ${ }^{64} \mathrm{Nel}$ 1940, Murillo La Greca rientra definitivamente a Recife, per affrescare la chiesa di Nossa Senhora da Penha (1869-82), che era stata progettata e costruita in stile eclettico con fondamenti neoclassici da un missionario cappuccino, padre Francesco da Vicenza, avendo come modello di riferimento la chiesa di Santa Maria Maggiore in Roma. ${ }^{65}$

Infine, un'altra presenza italiana che si fa sentire nell'architettura è quella di Giacomo Palumbo. Si tratta della stessa persona alla quale viene affidato il nuovo piano urbanistico di Natal, cui si è già accennato. A Recife, Palumbo è autore del progetto neo-rinascimentale del Palazzo di Giustizia (1924-1930) e più tardi del progetto del Ponte Duarte Coelho (1943).

\section{e. A Maceiò}

A partire dal 1925, l'agente consolare italiano di Maceió è Antonio Gerbasi, un immigrato giunto nello Stato di Alagoas nel 1884 da Vibonati, un piccolo centro del basso Cilento (Salerno). Gerbasi è titolare di un grande emporio commerciale dal 1898. Commerci e botteghe artigianali impegnano tutti gli italiani della città. Numerose sono le calzolerie e le sartorie. ${ }^{66}$

\section{f. Ad Aracaju}

Sono davvero pochi gli italiani giunti ad Aracaju, ma importante è la loro presenza dal punto di vista qualitativo. ${ }^{67}$ Grande successo vi ottiene Nicola Mandarino, di Vibonati (Salerno), giunto ad Aracaju da Bahia agli inizi del Novecento. Egli ha una grande falegnameria meccanica, una fabbrica di sapone e un magazzino di tessuti, grazie ai quali entra a far parte dell'élite sergipense e della massoneria locale.

La Loja Maçônica Cotinguiba, fondata nel 1872, si segnala per lo spazio offerto agli stranieri, di cui sanziona l'integrazione, e finisce con l'essere il più moderno ed eclettico strumento di rappresentanza sociale dell'élite 


\section{Dôssie Imigração}

locale. ${ }^{68}$ Da questa postazione privilegiata, Mandarino favorisce l'arrivo ad Aracaju di una "missione artistica" italiana, che, a partire dal 1918, rinnova l'assetto e l'arredo urbano della città, dandole un volto moderno. ${ }^{69}$ Il principale costruttore di Aracaju diventa Federico Gentile, calabrese di Paola, e l'architetto più importante è Bellando Bellandi. Quest'ultimo realizza in stile eclettico la facciata del Palazzo del Governo, che diventa un modello estetico per le residenze private dell'oligarchia locale. Successivamente si sposterà a Salvador de Bahia, dove lavorerà prevalentemente come scultore. ${ }^{70}$

\section{g. A Salvador de Bahia}

Gli italiani sono presenti a Salvador in forma organizzata già negli anni Sessanta dell'Ottocento (risale al 1861 la nascita di una Sociedade Italiana de Recreio e Beneficiência). Verso la fine del decennio successivo giungono i primi immigrati provenienti da Trecchina, che abbiamo già visto prevalenti nella comunità italiana di Recife. I trecchinesi danno vita alla colonia italiana di Jequié, un centro dell'interno dello Stato di Bahia, a circa 350 chilometri da Salvador. All'inizio del nuovo secolo, nella Baixa dos Sapateiros e nelle strade vicine del centro storico della capitale operano molti artigiani e commercianti italiani. Sono in tutto circa cinquecento immigrati, provenienti per lo più da Trecchina, dal vicino centro calabrese di Laino Borgo e da altri paesi posti al confine tra Basilicata e Calabria. La Pastelaria Triunfo, all'angolo tra la Baixa dos Sapateiros e la Ladeira da Saúde, è abituale luogo di ritrovo degli italiani. Alcuni di essi sono attivi anche nella piccola industria (paste alimentari, latticini, calzature, bevande). Negli anni Trenta, si conta la presenza di almeno due fabbriche italiane di paste alimentari: la "Brasil" e la "Progresso". Non mancano, inoltre, le sartorie e una fabbrica di letti. Una monografia di Thales de Azevedo ha registrato l'esistenza di 1.500 italiani nel 1920 e ha censito la presenza di 250 cognomi italiani a Bahia. ${ }^{71}$

I fratelli Scaldaferri esportano caffe, cacao e tabacco. A questo gruppo familiare appartiene Sante Scaldaferri (Salvador, 1928), un artista affermato a livello nazionale nel secondo Novecento brasiliano. Ma il suo non è un caso isolato di presenza culturale di ascendenza italiana. Negli anni Trenta, ad esempio, si registrava la curiosa presenza di una Barbearia Futurista, in omaggio al movimento d'avanguardia fondato da Marinetti. ${ }^{72}$ E già dal 1903 operava a Salvador lo scultore italiano Pasquale De Chirico, lucano di Venosa e allievo del verista napoletano Achille D’Orsi. Dopo dieci anni di lavoro a São Paulo, dove aveva aperto una fonderia, De Chirico, su 
invito dell'ingegnere Teodoro Sampaio (importante intellettuale baiano, noto in tutto il Brasile), si era trasferito a Salvador, dove avrebbe vissuto e operato per un quarantennio. Suoi sono i principali monumenti scultorei della città. ${ }^{73}$ Lo stesso Carlos Chiacchio, il principale animatore della cultura letteraria a Salvador nella prima metà del Novecento, è di chiare origini italiane. ${ }^{74}$

Inoltre, nel 1913, si era trasferito a Salvador l'architetto nomade Filinto Santoro, dopo aver abbandonato Belém. ${ }^{75}$ Per dieci anni circa, Santoro realizza edifici pubblici di notevole importanza, partecipando con un ruolo primo piano alla modernizzazione urbana dell'antica capitale del Brasile, la quale vive un processo di trasformazione che assume ritmi assai sostenuti e aspetti radicali e controversi negli anni del governo di José Joaquim Seabra (1912-16). Tra gli interventi di Santoro si segnala una Caserma dei Pompieri, costruita lungo la Baixa dos Sapateiros, che nell'idea progettuale s'ispira visibilmente e senza alcuna mediazione culturale ed estetica al Palazzo Pubblico di Siena.

Stabilite buone relazioni con i governatori dello Stato di Bahia, Santoro riceve anche l'incarico di ampliare e ristrutturare importanti edifici pubblici: il Mercado Modelo, il Palácio da Aclamação, residenza dei governatori, e, in collaborazione con Giulio Conti e Arlindo Fragoso, il Palácio Rio Branco, sede del governo. Nel 1918-19, Santoro realizza anche un progetto di natura privata: il cinema-teatro Kursaal-Babiano, esempio tardo di art nouveau, con una sala da 1.200 posti, all'avanguardia dal punto di vista tecnologico, che sarà rimaneggiato negli anni Cinquanta e infine distrutto e sostituito da un cinema-teatro progettato da Lina Bo Bardi nel 1986. ${ }^{76}$

Prima di lasciare Salvador, agli inizi degli anni Venti, l'ingegnere Santoro mise mano ad un ultimo progetto: l'Avenida Oceanica, che, terminando sul Farol da Barra, proteso sull'Oceano, rappresenta in qualche modo il suo commiato dal Brasile, prima di riattraversare per l'ultima volta l'Atlantico. Si conclude così una vicenda professionale, che può considerarsi emblematica della presenza culturale e artistica italiana al tempo della grande emigrazione transoceanica, con la quale peraltro Santoro è entrato sistematicamente in relazione. Una presenza pervasiva, quella degli architetti e degli artisti italiani, che ha attraversato l'intero Brasile, da Rio e da São Paulo alle regioni e alle città più periferiche, facendosi veicolo e filtro della cultura artistica europea tra Otto e Novecento. 
Notas e Referências

1 Vittorio CAPPELLI. Storie di italiani nelle altre Americhe. Bolivia, Brasile, Colombia, Guatemala e Venezuela; RUBBETTINO, Soveria MANNELLi, 2009; Id. "Immigrazione e urbanizzazione. La presenza degli italiani nelle 'altre Americhe”". Passato e Presente, vol. XXV, n. 71, 2007, pp. 21-44 (traduzione portoghese: "A propósito de imigração e urbanização: correntes imigratórias da Itália meridional às 'outras Américas”'. Estudos Ibero-Americanos, vol. XXXIII, n. 1, 2007, pp. 7-37).

2 Vittorio CAPPELLI. "Regioni migratorie e regioni politico-amministrative. L'emigrazione verso le 'altre Americhe' da un territorio di frontiera calabrolucano-campano". Archivio Storico dell'Emigrazione Italiana, vol. 3, n. 1, 2007, pp. 55-66.

3 Márcio SOUZA. História da Amazônia. Editora Valer, 2009, p. 236.

4 Non dovrebbero esserci più dubbi, alla luce di questa come di altre analoghe indagini, sul ruolo decisivo dell'Italia e degli italiani nella diffusione dell'eclettismo e dell' art nouveau in Brasile, soprattutto in campo architettonico. Su questo argomento cfr. anche: Denis ROLLAND. A crise do modelo francês. A França e a América Latina. Cultura, politica e identidade. Brasília: Editora UNB, 2005.

5 Jussara da Silveira DERENJI. Arquitetura nortista. A presença italiana no início do século XX, Manaus: SEC, 1998; Benedito NUNES e Milton HATOUN. Crônica de duas cidades. Belém e Manaus. Belém: Secult, 2006; Vittorio CAPPELLI. "Architetti e costruttori italiani nelle città brasiliane (e altrove) tra XIX e XX secolo". In: Atti del "Simpósio Nacional de História Cultural. Sensibilidades e sociabilidades”. Goiânia, 13-17 ottobre 2008 (in corso di stampa).

6 Monografia dei lavori eseguiti dalla ditta Antonio Jannuzzi e Fratelli, Architetti costruttori in Rio de Janeiro. Brasile: Stab. Tipo-litografico Cartoleria Maglia, Milano, s.d. (1905?).

7 Jussara da Silveira DERENJI. Arquitetura nortista. A presença italiana no início do século XX, op. cit.; Otoni MESQUITA. Manaus. História e arquitetura (1852-1910). Manaus: Editora Valer, 2006.

8 Vincenzo Scirchio, cognato di Michelangelo Jannuzzi, un altro fratello di Antonio, è originario di Castelluccio (Potenza). Il 10 novembre 1899 inviò un telegramma alla Voce d'Italia di Rio, che lo pubblicò e lo commentò con entusiasmo il giorno successivo.

9 Cf. Monografia dei lavori eseguiti dalla ditta Antonio Jannuzzi e Fratelli, op. cit. Le vittime sono da attribuirsi, verosimilmente, alla congiunta durezza del clima amazzonico e del lavoro. 
10 Ne dà notizia La Voce d'Italia di Rio de Janeiro l'8 gennaio 1900: “Col vapore Planeta proveniente dal Nord, ritornava alla capitale fluminense l'illustre connazionale nostro cav. Antonio Jannuzzi, reduce da quel lontano paese che si presenta come un nuovo Eldorado ai popoli civili e dove fu chiamato da un Governo per gettare la prima pietra" (del) "superbo, grande, splendido edifizio destinato all'opera umanitaria della Casa di Misericordia che va a sorgere in Manaos per opera sua, con la contribuzione diretta del Governo di circa undici milioni”.

11 L'insegnamento di G. B. SANTORO al Giovane JANNUZZI è rammentato nella Monografia dei lavori eseguiti dalla ditta Antonio Jannuzzi e Fratelli, op. cit. Per l'adesione 'militante' alla Chiesa Valdese cfr. Corriere Calabrese, Catanzaro, 20 e 24 gennaio 1900, dove si descrive l'accoglienza tributata al teologo Teofilo Gay, pastore valdese in Napoli e massone, da parte di Giuseppe Jannuzzi (nel frattempo rientrato da Rio a Fuscaldo), con l'intervento anche dei giovani Giotto e Attilio Santoro (che, invece, di lì a poco ritroveremo a Manaus). Gay e Jannuzzi si recano poi anche a Nicastro, col pastore valdese di Falerna, Giacomo Maugeri, il quale si era formato da emigrante negli Usa, presso il Seminario Teologico di Princeton e nella comunità valdese di Germantown, presso Philadelphia, che era stata accolta e sostenuta dalla Chiesa Presbiteriana (molteplici, dunque, i nessi tra migrazioni e protestantesimo valdese). Cf. Mario Folino GALLO e Vittoria BUTERA (a cura di). Falerna e la Comunità Evangelica. Padova: Panda Edizioni, 2009.

12 La Società Italiana di Beneficienza era nata addirittura nel 1854, per iniziativa di Teresa Cristina di Borbone, figlia del re delle Due Sicilie Francesco I, divenuta sposa dell'imperatore Dom Pedro II nel 1842. Si noti la larghezza di vedute di una Borbone di Napoli, che denomina come 'italiana' una società di beneficienza concepita ben prima dell'Unità della Penisola.

13 Nel 1895 viene costituita la Loggia Capitolare Massonica Fratellanza Italiana. Ripetute e sontuose erano in quegli anni le celebrazioni del XX Settembre tipico evento delle manifestazioni politiche della massoneria, in memoria della "breccia di Porta Pia" - alle quali partecipavano a migliaia gli esponenti della comunità italiana. A fianco ai fratelli Jannuzzi, vi intervenivano i fratelli Aleardo e Filinto Santoro, in qualità di organizzatori. Cf., a puro titolo esemplificativo, gli echi e i commenti apparsi sulla stampa italiana di Rio nel 1891 e nel 1892: "Il XX Settembre a Rio de Janeiro", in L'Indipendente, 24 settembre 1891; "Il XX Settembre a Rio de Janeiro". Il Bersagliere, 21-22 settembre 1891; "Il XX Settembre a Rio de Janeiro". In: L'Italia, 21 settembre 1892; "La festa de 20 Settembre”, In: La Voce del Popolo, 24 settembre 1892.

14 Su questo argomento, il documento fondamentale è: Marc FERREZ. O Álbum da Avenida Central (1903-1906). Rio de Janeiro: João Fortes Engenharia, 1982. 


\section{Dôssie Imigração}

15 Cf. Associazione Nazionale Ingegneri e Architetti Italiani, Sezione di Napoli, L'opera dell'ing.re Filinto Santoro al Brasile, Tipo-Editrice Meridionale Anonima, Napoli, 1923; Jussara da Silveira DERENJI. Arquitetura nortista. A presença italiana no início do século XX, op. cit.; Otoni MESQUITA. Manaus. História e arquitetura (1852-1910). Op.cit.

16 Domenico De Angelis (1853-1900) è un esponente di primo piano della celebre Accademia di San Luca in Roma. Uno dei pittori che lavorano con lui in Amazzonia è Giovanni Capranesi (1852-1921), che al termine della sua vita sarà anche presidente dell'Accademia di San Luca. Cfr. Jussara da Silveira DERENJI, op. cit.; Otoni MESQUITA, op. cit.; Vittorio CAPPELLI. Immigrazione e urbanizzazione. La presenza degli italiani nelle 'altre Americhe', op.cit.

17 Jussara da Silveira DERENJI, op. cit.; Otoni MESQUITA, op. cit.

18 Cfr. Elson FARIAS. Claudio Santoro, cantor do sol e da paz. Manaus: Editora Valer, 2009.

19 Márcio SOUZA. História da Amazônia, op.cit.

20 Su questi temi, cfr.: Leno José Barata SOUZA. "Evas, vadios e moleque: outras histórias da Belle Époque Barê"; Maria Luiza Ugarte PINHEIRO. "Nos meandros da cidade: cotidiano e trabalho na Manaus da borracha”; Paulo MARREIRO. "História e ação social: moradia e ameaça urbana"; tutti in Canoa do Tempo. Revista do Programa de Pós-Graduação em História da Universidade Federal do Amazonas, Manaus, n. 1, 2007. Cf. anche: Maria Luiza Ugarte PINHEIRO. A cidade sobre os ombros. Trabalho e conflito no Porto de Manaus, 1899-1925. Manaus: Edua, 2003.

21 Gregorio RONCA. "Dalle Antille alle Guyane e all'Amazzonia”. Rivista Marittima, Roma, 1908 (http://www.solofrastorica.it/amazzoniaviaggio.htm); Alfredo CUSANO. Italia d'oltremare. Impressioni e ricordi dei miei cinque anni in Brasile. Milano: Stabilimento Tipografico Enrico Reggiani, 1911.

22 Cf. Vittorio CAPPELLI. "Italiani in Amazzonia". In: Storie di italiani nelle altre Americhe. Op.cit.

23 Francesco Saverio NITTI, Scritti sulla questione meridionale, vol. IV,. Inchiesta sulle condizioni dei contadini in Basilicata e in Calabria (1910), t. II, Laterza, Bari, 1968, p. 282.

24 Alfredo CUSANO. Italia d'oltremare. Impressioni e ricordi dei miei cinque anni in Brasile, op. cit.

25 Indicador illustrado do Estado do Amazonas, Courrier \& Billiter Editores, Manaos, 1910.

26 Alfredo CUSANO. Italia d'oltremare, op. cit.

27 Giovanni BONACCI. Il Brasile e l'emigrazione italiana (1920). Roma: Vittorio Bonacci Editore, s.d. 
Brasile. Rassegna della vita e delle opere della stirpe italiaca negli Stati del Nord brasiliano. Belém, Parà: Typ. da Livraria Gillet, 1932 (3a edizione).

29 Sia l'orologio che le campane sono custoditi oggi nell' ingresso di un ex convento che ospita il municipio di Castelluccio Inferiore. Cfr. Silvia AQUINO. La Basilicata e la Calabria nella "Grande Emigrazione". Castelluccesi e Moranesi in Brasile. Tesi di laurea specialistica in Discipline Economiche e Sociali per lo Sviluppo e la Cooperazione, Università della Calabria, a. a. 2008-2009. Ermenegildo ALIPRANDI e Virgilio MARTINI. Gli italiani nel Nord del Brasile, op. cit.

31 Cf. Vittorio CAPPELLI. "Immigrazione e urbanizzazione. La presenza degli italiani nelle 'altre Americhe1'”, op. cit.

32 Cf. O Estado do Amazonas (Brasil). Genova: Stab. Tip. Montorfano, 1899; Vittorio CAPPELLI, "Immigrazione e urbanizzazione"m op. cit.; Id. "Italiani in Amazzonia”. IN: Storie di italiani nelle altre Americhe, op. cit.

33 Le castanhas do pará sono generalmente conosciute in Italia come "noci brasiliane"; il pirarucu è un enorme pesce d'acqua dolce, noto anche come o bacalhau da Amazônia.

34 Ermenegildo ALIPRANDI e Virgilio MARTINI. Gli italiani nel Nord del Brasile, op. cit.; sulla presenza italiana nel basso corso del Rio delle Amazzoni, cf. Marília Ferreira EMMI. Italianos na Amazônia. (1870-1950). Pioneirismo econômico e identidade. Belém, Pará: Edufpa, 2008.

Questa circostanza ribadisce il legame tra questa tipologia migratoria italiana, la massoneria e le confessioni protestanti, come si è già osservato a proposito dei Jannuzzi e dei Santoro. Per il protestantesimo, si riveda la nota n. 10. Per l'inventario dell'architettura di Óbidos cf. Jussara da Silveira DERENJI. Óbidos, Caderno de Arquitetura, 1, Belém, Pará: Ufpa, 1987. La figlia primogenita della signora Marietta, Rachel Peluso (Santarém, 1908São Paulo, 2005), sarà compositrice, strumentista e cantante. Nel 1945, fonderà, a São Paulo, l'Instituto Musical Padre José Maurício e lo dirigerà per decenni assieme alla sorella minore Gioconda. Cf. Vicente SALLES. Música e músicos do Pará, Conselho Estadual de Cultura, Belém, 1970; Vicente Malheiros da FONSECA. Rachel Peluso (http://cantodevarzea.blogspot.com/2009/06/rachelpeluso.html); Vittorio CAPPELLI. Immigrazione e urbanizzazione. La presenza degli italiani nelle 'altre Americhe'. Op.cit.

Ermenegildo ALIPRANDI e Virgilio MARTINI. Gli italiani nel Nord del Brasile, op. cit.; cf. anche la ricostruzione di questa piccola saga familiare. In: História da imigração italiana no Brasil. $1^{\circ}$ Centenário dos Calliari Parente no 
Brasil, 1870-1970 [tre fogli sciolti a stampa, senza data e luogo; presumibilmente: Belém, 1970].

39 Su Landi si veda ora l'accuratissimo sito web: Giuseppe Antonio Landi: il Bibiena dell'Equatore, a cura di Maria Cristina Turchi (http://www.forumlandi.ufpa.br/ index.html).

40 Jussara da Silveira DERENJI. Arquitetura nortista. A presença italiana no início do século XX, op. cit.; Otoni MESQUITA. Manaus. História e arquitetura (18521910), op. cit.; Vittorio CAPPELLI. Immigrazione e urbanizzazione. La presenza degli italiani nelle 'altre Americhe', op. cit.

41 Benedito NUNES e Milton HATOUM. Crônica de duas cidades. Belém e Manaus. Belém: Secult, 2006.; Marcelo GRUMAN. "A prostitução judaica no inicio do século XX: desafio à construção de uma indentidade étnica positiva no Brasil". Campos. Revista de Antropologia Social, n. 7, 1, 2006; Lená Medeiros de MENEZES. "Facetas marginais do sonho de civilização: imigração francesa e prostituição no Brasil (1816-1930)”. In :Laurent VIDAL e Tania Regina de LUCA (a cura di). Franceses no Brasil. Séculos XIX-XX. São Paulo: Editora Unesp, 2009.

42 Otoni MESQUITA. Manaus. História e Arquitetura (1852-1950), op. cit., pp. $146-47$.

43 Jussara da Silveira DERENJI. Ilusão e cor. Pintura de interiores na Arquitetura de Belém, Secult, Belém, 2004, p. 27.

44 Jussara da Silveira Derenji, Arquitetura nortista, cit. pp. 158-161.

45 Cf. Associazione Nazionale Ingegneri e Architetti Italiani, Sezione di Napoli, L'opera dell'ing. re Filinto Santoro al Brasile, op. cit.

46 Id. Ibidem.

47 Id. Ibidem. Per un giudizio critico su questo manufatto. Cf. Jussara da Silveira DERENJI. Arquitetura nortista, op. cit., pp. 186-190.

48 Alfredo CUSANO. Italia d'oltremare. Impressioni e ricordi dei miei cinque anni in Brasile. Op. cit.

49 Marília Ferreira EMMI. Italianos na Amazônia (1870-1950). Pioneirismo econômico e identidade, op. cit.

50 Ermenegildo ALIPRANDI e Virgilio MARTINI. Gli italiani nel Nord del Brasile. Op. cit.

51 Id. Ibidem; Vicente SALLES. Música e músicos do Pará. Op. cit.; Vincenzo CERNICCHIARO. Storia della musica nel Brasile: dai tempi coloniali ai giorni nostri, 1549-1925. Milano: Fratelli Riccioni, 1926.

52 Tatiana FERREIRA. Pesquisa revela negritude na música e na poesia paraenses (http://www.ufpa.br/beiradorio/arquivo/beira14/noticias/noticia8.htm); Lenora BRITO. Arte no TeatrodaPaz (http://theatrodapaz.com.br/web index.php?option=com_content\&task=view\&id=21 \&Itemid=36). 
Ermenegildo ALIPRANDI e Virgilio MARTINI. Gli italiani nel Nord del Brasile, op. cit..

Marcello CARMAGNANI. L'altro Occidente. L'America Latina dall'invasione europea al nuovo millennio. Torino: Einaudi, 2003, pp. 270-73.

\section{italiani ai quali viene affidata, tra Otto e Novecento, la Chiesa di N. S. da Penha.} La missione cappuccina italiana a Recife, che aveva avuto inizio addirittura nel 1710, dirigeva tutte le missioni del nord del Brasile. Cfr. Ermenegildo Aliprandi e Virgilio Martini, Gli italiani nel Nord del Brasile, cit.; sull' argomento cf. pure: Lúcia Noya GALVÃO e Salomé RATIS. Da religiosidade canônica à popular: a Basilica da Penha do Recife-Pernambuco (htpp://www.revistas.uepg. br/ index.php?journal=folkcom \&page ( $=$ article \&op $=$ viewFile\&path\%5B\%5D=489\&path\%5B\%5D=315). Ermenegildo ALIPRANDI e Virgilio MARTINI. Gli italiani nel Nord del Brasile, op. cit. Vittorio CAPPELLI. Immigrazione e urbanizzazione, op. cit. Cfr. Luis Antônio BARRETO. Estrangeiros em Aracaju, 29 aprile e 3, 7, 9, 12 maggio 2005 (http://www.infonet.com.br/luisantoniobarreto). Ne fanno parte Bellando Bellandi (architetto e scultore), Oreste Gatti (pittore), Bruno Cercelli. (scultore e decoratore), Raffaele Alfano (scalpellino). Cf. Vittorio CAPPELLI, Immigrazione e urbanizzazione. Op.cit. 
70 È suo un monumento a Ruy Barbosa (1932), nella cittadina di São Felix (Bahia).

71 Cfr. Thales de AZEVEDO. Italianos na Bahia e outros temas. Salvador: Empresa Gráfica da Bahia, 1989; Vittorio CAPPELLI. Immigrazione e urbanizzazione, op. cit.

72 Id. Ibidem.

73 Si possono qui ricordare la statua del poeta Castro Alves (1923), due grandi statue del Cristo (1920 e 1942), il gruppo scultoreo in onore del Visconde de Cairu, economista e intellettuale baiano vissuto tra il XVIII e il XIX secolo (1923), quattro lavori realizzati per gli interni del Palácio Rio Branco (1919), il monumento al portoghese dom Fernandes Sardinha (1944), primo vescovo del Brasile, divorato dagli indios antropofagi caetés in Alagoas, nel 1556. Cf. Juciara Maria Nogueira BARBOSA. Descompasso: como e porque o modernismo tardou a chegar na Bahia (http://www.cult.ufba.br/enecult2009/19289.pdf); Ubaldo Marques PORTO FILHO. Pasquale, o escultor (http://www.acirv.org/hp/ revista.asp?id=24\&tipo=geral)

74 Carlos CHIACCHIO (Januária, 1884-Salvador, 1947), creatore, tra l'altro, della rivista letteraria Arco \& Flexa nel 1928, era figlio di due immigrati italiani di evidenti origini meridionali (Chiacchio e Lavorato sono i loro cognomi). Cf. http://www.blogdogutemberg.blogspot.com/2006/06/carlos-chiacchio.html; Vittorio CAPPELLI. Immigrazione e urbanizzazione, op. cit.; Angelo Barroso Costa SOARES. Academia dos Rebeldes: modernismo à moda baiana. Dissertação de pós-graduação, Bahia, Universidade Estadual de Feira de Santana, 2005.

75 Cf. Associazione Nazionale Ingegneri e Architetti Italiani, Sezione di Napoli. L'opera dell'ing. re Filinto Santoro al Brasile, op. cit.

76 Vittorio CAPPELLI. Storie di italiani nelle altre Americhe. Bolivia, Brasile, Colombia, Guatemala e Venezuela. Rubbettino: Soveria Mannelli, 2009; Id. "Immigrazione e urbanizzazione. La presenza degli italiani nelle 'altre Americhe”. Passato e Presente, vol. XXV, n. 71, 2007, pp. 21-44 (traduzione portoghese: "A propósito de imigração e urbanização: correntes imigratórias da Itália meridional às 'outras Américas'”. Estudos Ibero-Americanos, vol. XXXIII, n. 1, 2007, pp. 7-37). 